\title{
An overview of heart failure in low- and middle-income countries
}

\author{
Valirie N. Agbor ${ }^{1,2}$, Ntobeko A. B. Ntusi ${ }^{3,4,5}$, Jean Jacques Noubiap ${ }^{3,6}$ \\ ${ }^{1}$ Ibal Sub-Divisional Hospital, Oku, Northwest Region, Cameroon; ${ }^{2}$ Department of Clinical Research, Health Education and Research Organization \\ (HERO), Buea, Cameroon; ${ }^{3}$ Division of Cardiology, Department of Medicine, University of Cape Town and Groote Schuur Hospital, Cape Town, \\ South Africa; ${ }^{4}$ Cape Universities Body Imaging Center, University of Cape Town, Cape Town, South Africa; ${ }^{5}$ Hatter Institute for Cardiovascular \\ Research in Africa, Department of Medicine, University of Cape Town, Cape Town, South Africa; ${ }^{6}$ Centre for Heart Rhythm Disorders, South \\ Australian Health and Medical Research Institute (SAHMRI), University of Adelaide and Royal Adelaide Hospital, Adelaide, Australia \\ Contributions: (I) Conception and design: All authors; (II) Administrative support: JJ Noubiap; (III) Provision of study materials or patients: None; \\ (IV) Collection and assembly of data: VN Agbor, JJ Noubiap; (V) Data analysis and interpretation: VN Agbor, JJ Noubiap; (VI) Manuscript writing: \\ All authors; (VII) Final approval of manuscript: All authors. \\ Correspondence to: Jean Jacques Noubiap. Department of Medicine, University of Cape Town and Groote Schuur Hospital, 7925 Observatory, Cape \\ Town, South Africa. Email: noubiapjj@yahoo.fr.
}

\begin{abstract}
Heart failure (HF) is a global public health concern with disproportionate socioeconomic, morbidity and mortality burden on low- and middle-income countries (LMICs). This review summarises contemporary data on the demographic and clinical characteristics, aetiologies, treatment, economic burden and outcomes of HF in LMICs. Patients with HF in LMICs are younger than those from high-income countries (HICs) and present at advanced stages of the disease. Hypertension, ischaemic heart disease (IHD), cardiomyopathy (CMO), and rheumatic heart disease (RHD) are the leading causes of HF in LMICs. The contribution of infectious diseases to HF remains prominent in many LMICs. Most health facilities in LMICs lack adequate diagnostic tools for HF, and the use of evidence-based medical and device therapies is suboptimal. Further, HF in LMICs is associated with prolonged hospital stay and high in-hospital and oneyear mortality. Finally, HF has profound economic impact on individual patients who, mostly, have no health insurance, and on societies where patients are young, comprising those who have the greatest potential to contribute to economic productivity.
\end{abstract}

Keywords: Heart failure (HF); aetiologies; mortality; management; low- and middle-income countries (LMICs)

Submitted Jun 10, 2019. Accepted for publication Aug 06, 2019.

doi: $10.21037 / \mathrm{cdt} .2019 .08 .03$

View this article at: http://dx.doi.org/10.21037/cdt.2019.08.03

\section{Introduction}

Heart failure (HF) is a major global health concern plaguing high-income countries (HICs) and low- and middleincome countries (LMICs) alike, and affects approximately 26 million people worldwide. HF is associated with substantial increased morbidity, mortality, poor quality of life and a significant economic burden on national healthcare systems (1-3). The prevalence of HF increases with age and is over nine fold higher among those 65 years or older compared to younger age groups $(4,5)$. In LMICs, available data suggest that HF is associated with high morbidity and mortality, prolonged hospitalisation and consequently carries a high societal burden $(2,3,6)$. The increasing burden of HF in LMICs is driven by an epidemiological transition and a surge in the prevalence of aetiological factors such as hypertension, diabetes mellitus, dyslipidaemia or obesity and lifestyle changes characterised by decreased physical activity, increased alcohol intake and smoking (7). Cardiovascular diseases (CVD), including HF, are major problems for health systems in LMICs facing a high burden of infectious diseases and are less prepared to cope with the double disease burden from communicable and non-communicable diseases (NCDs) (8).

Until recently, there has been a dearth of information on the epidemiology of HF in LMICs. Most data have come 
from hospital-based studies and there has been a paucity of data from population-based studies. Significant efforts have been made to reduce this knowledge gap. The growing body of evidence on HF in LMICs is revealing marked difference in causes, clinical characteristics, management and outcomes of HF in these countries compared to HICs (9). Hence, the current review summarises recent data on $\mathrm{HF}$ in LMICs in order to inform clinical practice, develop adequate knowledge translation strategies and help identify research gaps that will enable future research and collaborations. Specifically, this review recapitulates data on the aetiology, clinical characteristics, management and outcomes of HF in LMICs, as well as its economic burden.

\section{Clinical characteristics and aetiologies of HF in LMICs}

Patients with HF in LMICs are likely to be younger with a mean/median age of 63 years and ranging from 52 years in Africa to 70 years in South America, compared to a mean/ median age of 70 years in those from HICs $(3,6,10-12)$. Indeed, the mean age at admission for HF increases with country economic index; insured and financially stable patients with CVD are likely to have their condition better managed and controlled and will only develop terminal complications at an advanced age (6). The younger age of HF patients in LMICs reflects failure of health systems to detect and control risk factors for HF, in particular, hypertension, diabetes mellitus, dyslipidaemia, smoking and excessive alcohol intake. Also, the significant contribution of infections that lead to rheumatic heart disease (RHD), tuberculous pericarditis and constrictive pericarditis and human immunodeficiency virus-associated (HIV-associated) CVD could explain the younger age of persons living with HF in LMICs compared to HICs, where these condition are much less prevalent $(3,6,11)$. Overall, males seem to be the more affected, even though this is inconsistent across studies especially those from Africa $(6,11,13)$.

There is a significant variability in the severity of $\mathrm{HF}$ at presentation across different LMICs and regions. For instance, about $30.1-56.4 \%$ of patients present with advanced HF [New York Heart Association (NYHA) classes III and IV] at the time of diagnosis, with Africa and Asia depicting the more severe phenotypes at diagnosis (2). Africa and Asia are the two regions with the lowest rate of health and medication insurance and educational attainment (11). Social inequality limiting access to medication and healthcare, and unavailability or poor access to diagnostic tools such as electrocardiography (ECG), X-rays, echocardiography, computed tomography, cardiovascular magnetic resonance, invasive angiography and scintigraphic techniques contributes to poor cardiovascular outcomes. Further, lack of evidence-based therapies (drugs, devices and surgical interventions), limited financial, infrastructural and human resources necessary for screening and diagnosis of $\mathrm{HF}$ and ineffective primary and secondary prevention against CVD also contribute to the observed disparities between HF outcomes in LMICs and HICs $(3,14)$. In addition, socioeconomic factors add to high rates of rehospitalisation for HF in Africa and Asia $(3,11,13)$.

The four leading causes of HF in LMICs are ischemic heart disease, hypertensive heart disease (HHD), cardiomyopathies (CMOs) and RHD (Table 1). Of note, IHD which is the leading cause of HF in most LMICs is far less prevalent in Africa, where hypertension, CMOs and RHD are more common (15). The epidemiology of CVD in Africa, especially IHD and HF, is also confounded by unavailability of robust diagnostic tools such as angiography (3), and even of ECG, which should ideally be readily available in most settings in LMICs. For instance, the availability and affordability of ECG were reported to be low in both rural and urban health facilities in West Cameroon, Kenya and Uganda $(14,16)$. Therefore, IHD is likely to be underdiagnosed in Africa. Moreover, there is an anticipated increase in the prevalence of the condition on the continent especially with the rising prevalence of cardiovascular risk factors. Interestingly, the INTER-CHF study found that up to $20 \%$ of cases of HF in Africa were due to ischemic heart disease was which twice higher than most previous estimates; however, the study population comprised mostly of urban patients recruited from private hospitals who had similar cardiovascular risk factors as HF patients in $\operatorname{HICs}(3,6)$.

$\mathrm{HHD}$ is, by far, the leading cause of HF in Africa (prevalence of $12-52 \%$ ) (15). High prevalence of hypertension, associated with low rates of awareness, treatment and control all contribute to the frequency of HHD and its complications in the region (17-20). Increasingly, there are alarming reports of an escalation in the burden of hypertension in children and adolescents (21), and pregnant women in Africa (22). Besides African countries, many other LMICs bear a huge burden of HHD, including those in South America, Eastern Mediterranean and even Europe (Table 1).

CMOs (prevalence of 3-30\%) and RHD (prevalence of 11-25\%) are also important causes of HF in LMICs (Table 1). An important cause of acute and chronic HF, especially in 
Table 1 Major causes of HF in LMICs

\begin{tabular}{|c|c|c|c|c|}
\hline References & HHD (\%) & IHD (\%) & CMO (\%) & VHD (\%) \\
\hline Africa $(n=N R)$ & 46 & 8 & 24 & 18 \\
\hline Americas $(\mathrm{n}=\mathrm{NR})$ & 31 & 33 & 30 & 15 \\
\hline Eastern Mediterranean $(\mathrm{n}=\mathrm{NR})$ & 52 & 59 & 27 & 22 \\
\hline South East Asia ( $n=N R)$ & 12 & 45 & 14 & 19 \\
\hline Western Pacific $(n=N R)$ & 21 & 54 & 14 & 21 \\
\hline \multicolumn{5}{|l|}{ INTER-CHF, 2017 (LMICs) } \\
\hline Africa $(n=1,294)$ & 35 & 20 & 14 & 11 \\
\hline China $(n=991)$ & 14 & 45 & 15 & 11 \\
\hline South-East Asia $(n=811)$ & 15 & 56 & 3 & 12 \\
\hline Agbor et al. (3), 2018 (sub-Saharan Africa) $(n=10,098)$ & 39.2 & 7.2 & 22.7 & 13.8 \\
\hline China-HF, 2017 (China) $(n=5,556)$ & 50.9 & 49.6 & 16.0 & 15.5 \\
\hline
\end{tabular}

n, sample size; NR, not reported; LMICs, low- and middle-income countries; HHD, hypertensive heart disease; IHD, ischaemic heart disease; CMO, cardiomyopathy; VHD, valvular heart disease; HF, heart failure; INTER-CHF, International Congestive Heart Failure Study.

sub-Saharan Africa (SSA) is tuberculous pericarditis. Indeed, the HIV epidemic in the region has led to a marked increase in the incidence of tuberculous pericardial disease, including constrictive pericarditis (23). Although the prevalence of this condition as a cause of $\mathrm{HF}$ is relatively low $(3-13 \%$ of cases according to different series) (24), it is associated with very high mortality rates of up to $27.6 \%$ at 6 months (23).

The leading comorbidities associated with HF in LMICs include hypertension, valvulopathies (either primary or functional), diabetes mellitus, dyslipidaemia, chronic kidney disease and smoking $(11,12,25)$. Except for valvulopathies, the prevalence of these comorbid conditions is similar to HICs. In the International Congestive Heart Failure (INTER-CHF) study over half of HF patients from the Middle-East had diabetes mellitus compared to just about $17 \%$ of patients from Africa (11). The same study showed that presence of those comorbidities is significant predictors of adverse outcomes (2).

\section{Management of HF in LMICs}

Pharmacological treatment of HF with reduced ejection fraction (HFrEF) is based on the use of diuretics for the relief of congestion-related symptoms and of neurohormonal antagonists-beta-blockers, angiotensin converting enzyme inhibitors (ACEIs) or angiotensin receptor blockers (ARBs) and mineralocorticoid receptor antagonists (MRAs) - which have been demonstrated to improve survival (26). The use of medications for HF varies substantially across LMICs (Table 2). Loop diuretics are by far the most commonly used medications in these settings. In Africa, for instance, diuretics are among the most available and cheapest medications for CVD (16). On the other hand, the use of evidence-based medications such as ACEIs, ARBs, MRAs and beta-blockers is suboptimal. Beta-blockers are used in only about one in three patients with HF in LMICs $(3,27)$. Beta-1 selective blockers such as bisoprolol, carvedilol and metoprolol, with the evidencebasis for management of HF, have poor availability and affordability in many LMICs. Strategies to improve utilisation of these life-saving medications include costcontainment and promotion of generics, as well as education of physicians on good clinical practice in the management of HF. 
Table 2 Medications used for the management of HF in LMICs

\begin{tabular}{|c|c|c|c|c|c|}
\hline References & $\begin{array}{l}\text { Beta blocker } \\
\text { (\%) }\end{array}$ & $\begin{array}{c}\text { ACE inhibitor/ } \\
\text { angiotensin receptor } \\
\text { blocker }(\%)\end{array}$ & $\begin{array}{l}\text { Loop diuretic } \\
(\%)\end{array}$ & $\begin{array}{c}\text { Aldosterone } \\
\text { antagonists (\%) }\end{array}$ & $\begin{array}{l}\text { Digoxin } \\
(\%)\end{array}$ \\
\hline Africa $(n=N R)$ & 25 & 70 & 73 & 46 & NA \\
\hline Americas $(\mathrm{n}=\mathrm{NR})$ & 38 & 60 & 71 & 32 & NA \\
\hline Europe $(n=N R)$ & 29 & 64 & 71 & 41 & NA \\
\hline South East Asia ( $n=N R)$ & 26 & 31 & 65 & 15 & NA \\
\hline Western Pacific $(n=N R)$ & 27 & 47 & 57 & 17 & NA \\
\hline \multicolumn{6}{|l|}{ INTER-CHF, 2017 (LMICs) } \\
\hline India $(\mathrm{n}=858)$ & 57 & 68 & 81 & 47 & 25 \\
\hline China $(n=991)$ & 66 & 73 & 45 & 27 & 29 \\
\hline South-East Asia $(n=811)$ & 60 & 64 & 61 & 56 & 29 \\
\hline $\begin{array}{l}\text { Agbor et al. (3), } 2018 \text { (sub-Saharan } \\
\text { Africa) }(n=5,692)\end{array}$ & 31.4 & 75.6 & 81.6 & 51.5 & 31.5 \\
\hline China-HF, 2017 (China) $(n=5,556)$ & 25.6 & 27.0 & 30.1 & 26.6 & 17.8 \\
\hline
\end{tabular}

n, sample size; NA, not applicable; NR, not reported; HF, heart failure; LMICs, low- and middle-income countries; INTER-CHF, International Congestive Heart Failure Study.

Finally, most of the evidence on the pharmacological therapy of HF was generated in patients from HICs. Patients from some LMICs have been timidly represented in large scale until recently $(3,12,28)$. However, despite bearing the highest burden of $\mathrm{HF}$, data specific evidence from clinical trials on the effectiveness of evidencebased HF medications are deficient in Africa and most major clinical trials evaluating the effectiveness of these medications have not included participants from Africa (26,28-31). Since the characteristics of patients with HF in LMICs, especially those in Africa, vary and might therefore influence response to pharmacotherapy (32), it is vital to increase the involvement of patients from LMICs in international clinical trials of HF therapies in order to provide reliable evidence-based recommendations which are based on data derived from these populations. Hopefully, with recent regional and global HF registries including a good number of HF patients from Africa, the involvement of Africans in clinical trials of HF treatments will be easier than ever before.

\section{Outcomes of HF in LMICs}

HF has poor outcomes in LMICs, where it has been demonstrated to be associated with a prolonged hospital stay of about 10 days (range, 5-35 days) $(6,25)$ and inhospital mortality of $4-25 \%$, with the highest rates being reported in Africa, South America and Asia (3,6,12,25,33). Older age, presence of atrial fibrillation, previous history of stroke, chronic kidney disease and hyponatraemia have been associated with higher in-hospital mortality in patients with HF in Salvador (34). In the China-HF registry, the presence of acute myocardial infarction, infection, right bundle branch block, higher levels of total bilirubin and increased blood urea nitrogen were significant predictors of in-hospital mortality (33). Importantly, lower systolic blood pressure was associated with reduced in-hospital mortality, suggesting the importance of blood pressure 
control on HF outcomes (33). Despite the high prevalence of IHD in the China-HF registry, only $3.8 \%$ benefited from a percutaneous coronary intervention compared with at least $8 \%$ of patients in the ADHERE (United States), ATTEND (Japan), EHFS (Europe), and KorAHF (Korea) registries (33). Such a difference is likely explained by limited resources and specialists limiting the application of this lifesaving intervention in the management of acute coronary syndromes in LMICs such as China as compared to HICs. As mentioned above, ischemic heart disease is probably underdiagnosed and poorly managed in many LMICs, especially in Africa, due to poor access to adequate diagnostic and therapeutic interventions.

In the INTER-CHF study, the overall one-year mortality rate for HF in LMICs was $16.5 \%$ with Africa and India bearing over $50 \%$ of the disease burden (2). Older age, previous and current admission for HF, advanced disease (NYHA functional class III or IV), valvular disease, chronic kidney disease and chronic obstructive pulmonary disease were independent predictors of one-year mortality (3). Furthermore, patients in Africa, India and South-East Asia had higher mortality rates compared to those in South America (2). Patients on ACEIs/ARBs or digoxin had a lower mortality (2). Surprisingly, beta-blockers were not found to be associated with reduced mortality (2). This might be due to either the use of inappropriate class of betablockers or inadequate titration, or the period of follow-up was not long enough to reveal the effect of beta-blockers on mortality, or better still, the study design was not the best to study the effects of beta blockers on HF-related mortality and therefore suggests the need of a large scale randomised controlled trial to evaluate the effectiveness of beta blockers on HF-related morbidity and mortality.

HF-related mortality across various LMICs are most likely related to differences in the quality of health facilities, low socioeconomic status (35), clinical practice guidelines for the management of HF, the number or proportion of physicians, cardiologists or nurses involved in patient follow-up, and patients' accessibility to, affordability of, and adherence to HF treatment. However, most registries in LMICs are yet to evaluate these claims. In the China-HF registry, for example, the investigators reported deviations of local practice guidelines with that of most western countries coupled with over- and underdosing of evidencebased medications (33). Of course, the determinants of mortality in LMICs are likely to be even more complex consisting an interplay between wealth, access to healthcare, quality of healthcare delivery, and presence of health infrastructures $(2,6)$.

\section{Economic burden of HF in LMICs}

With higher poverty rates, most LMICs are ill-prepared to control the fast-growing burden of chronic NCDs like $\mathrm{HF}$, which is likely to impede ongoing initiatives to curb poverty in LMICs, leading to a continuity in the vicious cycle of economic inequality and increasing burden of $\mathrm{HF}$ $(35,36)$. The global target in reducing CVD mortality by $25 \%$ by the year 2025 remains far-fetched in most LMICs primarily due to poor governance, insufficient allocation of public funds to the health sector, lack of evidence-based and ineffective delivery of healthcare including disease prevention, low levels of awareness, and absence of universal health coverage, among others (37-40).

In 2012, HF was associated with an overall cost of $\$ 15,130$ in LMICs, $90 \%$ of which was from indirect cost (healthcare expenditure owing to loss of productivity resulting from disability and death, welfare support and sickness benefits) (40). The trend is different in HICs where majority of the cost of HF was from direct spending on healthcare medication, physician visits and hospital services, highlighting the fact that most patients in LMICs encounter major difficulty in meeting the costs associated with $\mathrm{HF}$ and other CVDs (41). Eventually, poverty increases morbidity and mortality, loss of economic productivity and indirect costs on national systems.

The cost of inpatient care represents about $25-56 \%$ of the total direct cost of HF management $(12,42)$. With high costs of inpatient care for $\mathrm{HF}$, low health insurance rates and almost inexistent universal healthcare, it is most likely that majority of the patients in LMICs presenting with acute decompensated HF cannot afford management costs which consequently lead to higher rates of in-hospital mortality compared to HICs (10). Investing in the prevention of HF should be an utmost priority in HICs and LMICs alike, especially with the escalating global burden of CVD risk factors. Prevention in LMICs requires intensification of the already multidisciplinary collaborations between international organisations, governments, physicians and researchers, civil society and patient groups.

\section{Conclusions}

Recent data reveal the ugly face of HF in LMICs with significant disparities in sociodemographic and clinical profiles compared with patients in HICs. The four leading 
causes of HF in LMICs are HHD, IHD, RHD and CMOs. Infection remains an important contributor to the development of HF in LMICs. Patients from LMICs are younger, mostly uninsured and diagnosed as in patients at advanced stages of the disease. HF in LMICs is associated with higher in-hospital and one-year mortality rates compared with studies from HICs. The use of evidencebased medications for HF is suboptimal and should therefore be scaled up in resource-poor settings. There is an urgent need for international collaboration among key actors and for governments in LMICs to improve national health budgets and to prioritise CVD prevention. It is crucial to scale up the use of research on and the use of evidence-based medications such as ACEIs, ARBs, betablockers and MRAs and to invest in invasive management of HF. Finally, large scale cohort and national HF registries are warranted to improve our understanding of the epidemiology of HF in LMICs.

\section{Acknowledgments}

Funding: NABN gratefully acknowledges funding from the South African Medical Research Council, National Research Foundation, the Harry Crossley Foundation and the Lily and Ernst Hausmann Trust.

\section{Footnote}

Provenance and Peer Review: This article was commissioned by the Guest Editor (Ntobeko A. B. Ntusi) for the series "Cardiovascular Diseases in Low- and Middle-Income Countries" published in Cardiovascular Diagnosis and Therapy. The article was sent for external peer review organized by the Guest Editor and the editorial office.

Conflicts of Interest: All authors have completed the ICMJE uniform disclosure form (available at http://dx.doi. org/10.21037/cdt.2019.08.03). The series "Cardiovascular Diseases in Low-and Middle-Income Countries" was commissioned by the editorial office without any funding or sponsorship. NABN served as the unpaid Guest Editor of the series and acknowledges funding from the South African Medical Research Council, National Research Foundation, the Harry Crossley Foundation and the Lily and Ernst Hausmann Trust. The other authors have no other conflicts of interest to declare.

Ethical Statement: The authors are accountable for all aspects of the work in ensuring that questions related to the accuracy or integrity of any part of the work are appropriately investigated and resolved.

Open Access Statement: This is an Open Access article distributed in accordance with the Creative Commons Attribution-NonCommercial-NoDerivs 4.0 International License (CC BY-NC-ND 4.0), which permits the noncommercial replication and distribution of the article with the strict proviso that no changes or edits are made and the original work is properly cited (including links to both the formal publication through the relevant DOI and the license). See: https://creativecommons.org/licenses/by-nc-nd/4.0/.

\section{References}

1. Ambrosy AP, Fonarow GC, Butler J, et al. The global health and economic burden of hospitalizations for heart failure: lessons learned from hospitalized heart failure registries. J Am Coll Cardiol 2014;63:1123-33.

2. Dokainish H, Teo K, Zhu J, et al. Global mortality variations in patients with heart failure: results from the International Congestive Heart Failure (INTERCHF) prospective cohort study. Lancet Glob Health 2017;5:e665-72.

3. Agbor VN, Essouma M, Ntusi NAB, et al. Heart failure in sub-Saharan Africa: a contemporaneous systematic review and meta-analysis. Int J Cardiol 2018;257:207-15.

4. Lesyuk W, Kriza C, Kolominsky-Rabas P. Cost-of-illness studies in heart failure: a systematic review 2004-2016. BMC Cardiovasc Disord 2018;18:74.

5. Lee $\mathrm{H}, \mathrm{Oh} \mathrm{SH}$, Cho H, et al. Prevalence and socioeconomic burden of heart failure in an aging society of South Korea. BMC Cardiovasc Disord 2016;16:215.

6. Callender T, Woodward M, Roth G, et al. Heart failure care in low- and middle-income countries: a systematic review and meta-analysis. PLoS Med 2014;11:e1001699.

7. Noubiap JJ, Bigna JJ, Nansseu JR, et al. Prevalence of dyslipidaemia among adults in Africa: a systematic review and meta-analysis. Lancet Glob Health 2018;6:e998-1007.

8. GBD 2017 Causes of Death Collaborators. Global, regional, and national age-sex-specific mortality for 282 causes of death in 195 countries and territories, 1980-2017: a systematic analysis for the Global Burden of Disease Study 2017. Lancet 2018;392:1736-88.

9. Kraus S, Ogunbanjo G, Sliwa K, et al. Heart failure in sub-Saharan Africa: a clinical approach. S Afr Med J 2016;106:23-31. 
10. Dewan P, Rørth R, Jhund PS, et al. Income inequality and outcomes in heart failure: a global between-country analysis. JACC Heart Fail 2019;7:336-46.

11. Dokainish H, Teo K, Zhu J, et al. Heart failure in Africa, Asia, the Middle East and South America: the INTERCHF study. Int J Cardiol 2016;204:133-41.

12. Rajadurai J, Tse HF, Wang CH, et al. Understanding the epidemiology of heart failure to improve management practices: an Asia-Pacific perspective. J Card Fail 2017;23:327-39.

13. Damasceno A, Mayosi BM, Sani M, et al. The causes, treatment, and outcome of acute heart failure in 1006 Africans from 9 countries. Arch Intern Med 2012;172:1386-94.

14. Carlson S, Duber HC, Achan J, et al. Capacity for diagnosis and treatment of heart failure in sub-Saharan Africa. Heart 2017;103:1874-9.

15. Ntusi NB, Mayosi BM. Epidemiology of heart failure in sub-Saharan Africa. Expert Rev Cardiovasc Ther 2009;7:169-80.

16. Jingi AM, Noubiap JJ, Ewane Onana A, et al. Access to diagnostic tests and essential medicines for cardiovascular diseases and diabetes care: cost, availability and affordability in the West Region of Cameroon. PLoS One 2014;9:e111812.

17. Ataklte F, Erqou S, Kaptoge S, et al. Burden of undiagnosed hypertension in sub-Saharan Africa: a systematic review and meta-analysis. Hypertension 2015;65:291-8.

18. Noubiap JJ, Nansseu JR, Nkeck JR, et al. Prevalence of white coat and masked hypertension in Africa: a systematic review and meta-analysis. J Clin Hypertens (Greenwich) 2018. [Epub ahead of print].

19. Noubiap JJ, Nansseu JR, Nyaga UF, et al. Global prevalence of resistant hypertension: a meta-analysis of data from 3.2 million patients. Heart 2019;105:98-105.

20. Nansseu JR, Noubiap JJ, Mengnjo MK, et al. The highly neglected burden of resistant hypertension in Africa: a systematic review and meta-analysis. BMJ Open 2016;6:e011452.

21. Noubiap JJ, Essouma M, Bigna JJ, et al. Prevalence of elevated blood pressure in children and adolescents in Africa: a systematic review and meta-analysis. Lancet Public Health 2017;2:e375-86.

22. Noubiap JJ, Bigna JJ, Nyaga UF, et al. The burden of hypertensive disorders of pregnancy in Africa: a systematic review and meta-analysis. J Clin Hypertens (Greenwich) 2019;21:479-88.
23. Mayosi BM, Wiysonge CS, Ntsekhe M, et al. Mortality in patients treated for tuberculous pericarditis in sub-Saharan Africa. S Afr Med J 2008;98:36-40.

24. Noubiap JJ, Agbor VN, Ndoadoumgue AL, et al. Epidemiology of pericardial diseases in Africa: a systematic scoping review. Heart 2019;105:180-8.

25. Bocchi EA, Arias A, Verdejo H, et al. The reality of heart failure in Latin America. J Am Coll Cardiol 2013;62:949-58.

26. Ponikowski P, Voors AA, Anker SD, et al. 2016 ESC Guidelines for the diagnosis and treatment of acute and chronic heart failure: The Task Force for the diagnosis and treatment of acute and chronic heart failure of the European Society of Cardiology (ESC) developed with the special contribution of the Heart Failure Association (HFA) of the ESC. Eur Heart J 2016;37:2129-200.

27. Nyaga UF, Bigna JJ, Agbor VN, et al. Data on the epidemiology of heart failure in Sub-Saharan Africa. Data Brief 2018;17:1218-39.

28. Yancy CW, Jessup M, Bozkurt B, et al. 2017 ACC/ AHA/HFSA focused update of the 2013 ACCF/AHA Guideline for the management of heart failure: a report of the American College of Cardiology/American Heart Association Task Force on clinical practice guidelines and the heart failure society of America. Circulation 2017;136:e137-61.

29. Kristensen SL, Martinez F, Jhund PS, et al. Geographic variations in the PARADIGM-HF heart failure trial. Eur Heart J 2016;37:3167-74.

30. Blair JE, Zannad F, Konstam MA, et al. Continental differences in clinical characteristics, management, and outcomes in patients hospitalized with worsening heart failure results from the EVEREST (efficacy of vasopressin antagonism in heart failure: outcome study with tolvaptan) program. J Am Coll Cardiol 2008;52:1640-8.

31. Greene SJ, Fonarow GC, Solomon SD, et al. Global variation in clinical profile, management, and postdischarge outcomes among patients hospitalized for worsening chronic heart failure: findings from the ASTRONAUT trial. Eur J Heart Fail 2015;17:591-600.

32. Skrzynia C, Berg JS, Willis MS, et al. Genetics and heart failure: a concise guide for the clinician. Curr Cardiol Rev 2015;11:10-7.

33. Zhang Y, Zhang J, Butler J, et al. Contemporary epidemiology, management, and outcomes of patients hospitalized for heart failure in China: results from the China heart failure (China-HF) registry. J Card Fail 2017;23:868-75. 
34. Latado AL, Passos LC, Braga JC, et al. Predictors of inhospital lethality in patients with advanced heart failure. Arq Bras Cardiol 2006;87:185-92.

35. Rosengren A, Smyth A, Rangarajan S, et al. Socioeconomic status and risk of cardiovascular disease in 20 lowincome, middle-income, and high-income countries: the Prospective Urban Rural Epidemiologic (PURE) study. Lancet Glob Health 2019;7:e748-60.

36. World Health Organization. Global status report on noncommunicable diseases 2014. Available online: https://www.who.int/nmh/publications/ncd-statusreport-2014/en/

37. Roth GA, Nguyen G, Forouzanfar MH, et al. Estimates of global and regional premature cardiovascular mortality in 2025. Circulation 2015;132:1270-82.

38. Yusuf S, Wood D, Ralston J, et al. The World Heart

Cite this article as: Agbor VN, Ntusi NAB, Noubiap JJ. An overview of heart failure in low- and middle-income countries. Cardiovasc Diagn Ther 2020;10(2):244-251. doi: 10.21037/ cdt.2019.08.03
Federation's vision for worldwide cardiovascular disease prevention. Lancet 2015;386:399-402.

39. Gheorghe A, Griffiths U, Murphy A, et al. The economic burden of cardiovascular disease and hypertension in lowand middle-income countries: a systematic review. BMC Public Health 2018;18:975.

40. Cook C, Cole G, Asaria P, et al. The annual global economic burden of heart failure. Int J Cardiol 2014;171:368-76.

41. Wirtz VJ, Kaplan WA, Kwan GF, et al. Access to medications for cardiovascular diseases in low- and middleincome countries. Circulation 2016;133:2076-85.

42. Ogah OS, Stewart S, Onwujekwe OE, et al. Economic burden of heart failure: investigating outpatient and inpatient costs in Abeokuta, Southwest Nigeria. PLoS One 2014;9:e113032. 